\title{
Existence of FGFR1-5-HT1AR heteroreceptor complexes in hippocampal astrocytes. Putative link to 5-HT and FGF2 modulation of hippocampal gamma oscillations
}

Dr. Manuel Narvaez, Dr. Yuniesky Andrade-Talavera, D. Ramon Fores-Pons, Dr. Ismael Valladolid- Acebes, Dra. Pia Siegele, Dr. Alejandro Hernandez-Sosa, Dr. André Fisahn, Dr. Alexander López-Salas, Dr. Dasiel O. Borroto-Escuela

The majority of the fibroblast growth factor receptor 1-serotonin 1 A receptor (FGFR1-5-HT1AR) heterocomplexes in the hippocampus appeared to be located mainly in the neuronal networks and a relevant target for antidepressant drugs. Through a neurochemical and electrophysiological analysis it was therefore tested in the current study if astrocytic FGFR1-5HT1AR heterocomplexes also exist in hippocampus. They may modulate the structure and function of astroglia in the hippocampus leading to possible changes in the gamma oscillations. Localization of hippocampal FGFR1-5-HT1AR heterocomplexes in astrocytes was found using in situ proximity ligation assay combined with immunohistochemistry using glial fibrillary acidic protein (GFAP) immunoreactivity as a marker for astroglia. Acute i.c.v. treatment with 8-OHDPAT alone or together with basic fibroblast growth factor (FGF2) significantly increased FGFR15-HT1AR heterocomplexes in the GFAP positive cells, especially in the polymorphic layer of the dentate gyrus (PoDG) but also in the CA3 area upon combined treatment. No other hippocampal regions were studied. Also, structural plasticity changes were observed in the astrocytes, especially in the PoDG region, upon these pharmacological treatments. They may also be of relevance for enhancing the astroglial volume transmission with increased modulation of the neuronal networks in the regions studied. The effects of combined FGF2 and 5-HT agonist treatments on gamma oscillations point to a significant antagonistic interaction in astroglial FGFR1-5-HT1AR heterocomplexes that may contribute to counteraction of the 5-HT1ARmediated decrease of gamma oscillations. 